\title{
Access to Health Information: a Key for Better Health in the Knowledge Society
}

\author{
A. Geissbuhler \\ Department of Imaging and Medical Informatics, Geneva University Hospitals, Geneva
}

\begin{abstract}
Summary
Objectives: to illustrate how access to health information is a key determinant to the strengthening of health and healthcare systems, and highlight related current challenges of the evolving knowledge society.

Methods: reflections on some of the dimensions of access to health information, with a focus on current evolutions of the World-WideWeb, unresolved issues and research perspectives.

Conclusion: our knowledge society is growing, maturing, and bringing powerful tools that can improve the quality, safety, and efficiency of healthcare processes and help citizens manage their health. However, many obstacles and challenges are still facing us. These include our ability to manage information and knowledge rather than data, produce decision-support tools with contents and ergonomics that effectively change behaviors, orient information users in the ever-growing jungle of the social World-Wide-Web, learn how to make sense and enable knowledge discovery in the huge and increasingly connected biomedical data repositories, and bridge the digital divides that exist within communities and across the world.

\section{Keywords}

Information society, information retrieval, social web, semantic web, globalization, patient empowerment

Geissbuhler A, Kulikowski C, editors. IMIA Yearbook of Medical Informatic 2008. Methods Inf Med 2008; 47 Suppl 1: 20-1
\end{abstract}

Bringing the Right Information to the Right Person at the Right Time: Moore's Law vs Mooers' Law

One of the main promises of health informatics is to improve the decisionmaking and production processes of health and healthcare, by bringing the right information at the right person and at the right time. Information systems get better integrated, both within and across institutions, and technology brings ubiquitous access through mobile connectivity, and ever-fancier physical devices. As they become omnipresent, these tools tend to disappear and leave us with the non-technological challenges, for which Moore's law of digital technological progress does not apply: turning data into information and knowledge, formatting and delivering these so that behaviors actually change, solving the organizational issues that hinder the deployment of these tools, and understanding societal challenges that arise when making information available and transparent. In some of these instances, it is likely that another law, Mooers' Law of information retrieval $[1,2]$, would apply, given the potential burden of possessing information and having to deal with it. This is particularly true when considering the humongous amount of existing on-line information which overwhelms the human brain and challenges even the best computer-based information and knowledge management systems.

\section{An Informed Patient is Em- powered: Challenges from the Web 2.0}

Most internet users look for health and healthcare information on line, and many use the retrieved information to discuss or make decisions about the management of their care. Tools to help them appreciate the trustworthiness of what they find on-line have been developed for a decade [3]. With the advent of the social Web, or Web 2.0 [4], new ways of accessing, producing, and sharing information have appeared and, along with the development of Internetbased health and healthcare services, redefine even further the relationships between citizens, patients, care providers and regulators. The emergence of this new social movement also challenges deeper societal values such as privacy, and, in a broader sense, the interactions between the individual, communities, and society.

\section{Information-enabled Bio- medical Research at the Age of Web 3.0}

As the semantic Web, or Web 3.0 promises to connect various domains 
of knowledge, from molecules to populations, from physics to cognitive sciences, into a coherent, machineprocessable network $[5,6]$, biomedical research techniques and paradigms will have to be revisited. The massive amount of data generated by health systems becomes a mine for ontologydriven, automated knowledge extraction devices. This "info-bio convergence" not only enables in silico experimentation, it also connects molecular information to clinical information, facilitates biomedical research and surveillance from operational, live databases, and the feedback of newly acquired knowledge into the clinical decision- and production-processes, creating a virtuous circle for learning.

\section{Bridging the Many Digital Divides}

A knowledge society, dematerialized in essence, knows little borders, but reveals and exacerbates existing economical, educational, and social divides, within countries, and across the world. Gaps in access to computer hardware, to internet connectivity, and the ability to use these tools meaningfully are obvious. Furthermore, globalization also reveals tensions in what Dominique Wolton calls the "infernal triangle of culture, identity and communication" [7], and the potential destructive disconnects between the dominant emitters of information (i.e, the Western World) and the many other receivers. If economical development and education are keys to bridging these divides, a better understanding and inclusion of the needs and socio-cultural determinants of the "have-nots" are also essential.

\section{Conclusion}

Access to health information is rapidly evolving, with each generation of the World-Wide-Web bringing new tools and challenges, new answers and questions. The promises of these tools and technologies will only come true if we learn how to master the "soft stuff", which, the saying goes, is really the hard stuff.

\section{References}

1. Mooers CN. Mooers' Law; or why some retrieval systems are used and others are not. (Zator Technical Bulletin 136). Cambridge, MA: Zator Company; 1959.

2. Austin B. Mooers' Law: In and out of Context. Journal of the American Society for Information
Science and Technology 2001;52(8):607-609

3. Boyer C, Selby M, Appel RD. The Health On the Net Code of Conduct for medical and health web sites. Medinfo. 1998;:1163-6.

4. McLean R, Richard BH, Wardman JI. The effect of Web 2.0 on the future of medical practice and education: Darwikinian evolution or folksonomic revolution? MJA 2007;187:174-177

5. Berners-Lee T, Hendler J, Lassila O. The semantic web. a new form of web content that is meaningful to computers will unleash a revolution of new possibilities. Sci Am 2001: www.sciam.com/ article.cfm?articleID=00048144-10D2-1C7084A9809EC588EF21, last accessed on March 30, 2008.

6. Berners-Lee T, Hall W, Hendler J, Shadbolt N, Weitzner DJ. Creating a science of the Web. Science 2006;313(5788):769-71

7. Wolton D. L'autre mondialisation. Flammarion 2003, Paris. ISBN 978-2-08-210273-5

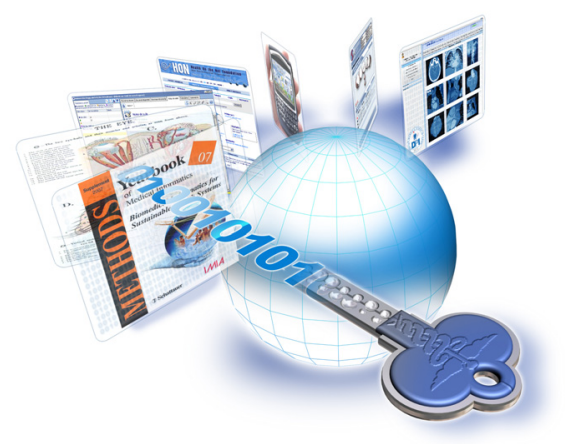

Yearbook cover illustration design by Constantin Sandru: a key unlocks the digital flow of multi-faceted, global health and biomedical information, from the scientific literature, the electronic patient record, the medical websites, and digital collaborative tools 